\title{
О СООТНОШЕНИИ МЕЖДУ АМПЛИТУДНЫМ, ЭФФЕКТИВНЫМ И СРЕДНИМ ЗНАЧЕНИЯМИ ЭЛЕКТРИЧЕСКОГО СИГНАЛА
}

\section{Л. и. волгин}

Интенсивность переменных напряжений можно характеризовать несколькими параметрами, определяющими величину электрического сигнала с точки зрения количественного воздействия в том или ином физнческом явлении или процессе. Основными параметрами, характеризующими интенсивность переменных напряжений, являются их

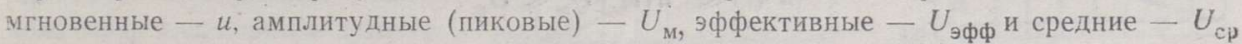
значения. Из перечисленных величин нанболее часто используется эффективное (действуюшее, среднеквадратичное) значение, определяюшее среднюю мощность электрического сигнала, в частности, его тепловой эффект.

Амплитудное, эффективное и среднее значения связаны между собой через коэффициенты амплитуды (пикфактор), формы (формфактор) и усреднения

$$
\begin{aligned}
& K_{\mathrm{a}}=\frac{U_{\mathrm{M}}}{U_{\text {эфф }}}, \\
& K_{\phi}=\frac{U_{э \phi \phi}}{U_{\mathrm{cp}}} \\
& K_{\mathrm{y}}=\frac{U_{\mathrm{M}}}{U_{\mathrm{cp}}} .
\end{aligned}
$$

Bсе три указанных коэффициента связаны соотношеннем

$$
K_{\mathrm{y}}=K_{\mathrm{a}} K_{\phi} .
$$

В силу известных причин в измерительной технике большое распространение по лучили вольтметры с неквадратичным законом детектирования, проградуированные в зффективных значениях синусоидального напряжения $\left(K_{\mathrm{a} 0}=\sqrt{2}, K_{\phi 0}=\frac{\pi}{2} \sqrt{2}\right)$. При использовании этих приборов для нзмерения эффективного значения напряжений (Э3Н) произвольной формы возникает дополнительная погрешность, обусловленная влиянием формы кривой измеряемого напряжения. Для вольтметров с линейным и пиковым детекторами эта погрешность определяется соответственно выражениями

$$
\begin{aligned}
& \delta_{\text {ср }}=\frac{K_{\phi 0}}{K_{\text {ф}}}-1, \\
& \delta_{\text {пик }}=\frac{K_{\mathrm{a}}}{K_{\mathrm{a} 0}}-1 .
\end{aligned}
$$


Нсследованию зависимости погрешности измерення ЭЗН от влияния формы крнвой при заданном неквадратичном законе детектирования посвящено довольно много работ. Использование полученных в этих работах результатов затрудняется тем, что при эксплуатации измерительных приборов необходимо предварнтельно знать велнчину $\boldsymbol{K}_{\phi}$ или $\boldsymbol{K}_{\mathrm{a}}$, а зачастую амплитудный и фазовый спектры измеряемого сигнала, что во многих случаях является затруднительным [1, 2].

Представляет интерес рассмотреть возможность определення велнчнны эффектнвного значения напряжений через произведение амплитудного и среднего значений. Введем обозначение

$$
K=\sqrt{\frac{K_{\mathrm{a}}}{K_{\phi}}}=\frac{V \overline{U_{\mathrm{m}} U_{\mathrm{cp}}}}{U_{\text {эфф }}}
$$

Из последнего соотношення получнм

$$
U_{9 \phi \Phi}^{2}=U_{\mathrm{cp}} \frac{U_{9 \mathrm{~s}}}{K^{2}}
$$

Рассмотрнм, в каких пределах может нменяться велнчнна $K$. Очевндно, что для электрического снгнала справедливо условне

$$
u^{2}(t) \leqslant|u(t)| U_{m} \leqslant U_{m}^{2} .
$$

Воспользовавшись правнлом интегрирования неравенств, можек записать

$$
\frac{1}{T} \int_{0}^{T} u^{2}(t) d t \leqslant \frac{U_{M}}{T} \int_{0}^{T}|u(t)| d t \leqslant U_{M}^{2} .
$$

После преобразованнй получнм

$$
1 \leqslant K^{2} \leqslant K_{\mathrm{a}}^{2}
$$

Так как значення

$$
K>0 \text { is } K_{a}>0
$$

To

$$
1 \leqslant K \leqslant K_{\mathrm{a}}
$$

С другой стороны, воспользовавшись теоремой о среднем [], для непрерывной функции можем записать

$$
K^{2}=\frac{\frac{U_{\mathrm{v}}}{T} \int_{0}^{T}|u(t)| d t}{\frac{\left|u\left(t_{k}\right)\right|}{T} \int_{0}^{T}|u(t)| d t},
$$

здесь $t_{k}$ есть неноторое значение, прннадлежащее промежутку $(0, T)$. Нз условня (13) найдем

$$
\frac{U_{\mathbf{u}}}{K^{2}}=\left|u\left(t_{k}\right)\right|
$$

Прн эrom

$$
U_{9 \phi \phi} \leqslant\left|u\left(t_{k}\right)\right| \leqslant U_{M} .
$$


Назовем величину $\left|u\left(t_{k}\right)\right|$ квазипиковым значением сигнала. Таким образом, согласно (8), квадрат эффективного значения равен произведению среднего и квазипи кового значений сигнала. При $K=1$ квадрат эффективного значения непосредственно равен произведению среднего и амплитудного значений сигнала, т. е. квазипиковое и амплитудное значения равны.

Из проведенного анализа следует, что при $K \simeq 1$

$$
U_{\ominus \phi \Phi} \simeq \sqrt{U_{\mathrm{cp}} U_{\mathrm{M}}} .
$$

При этом относительная погрешность определения эффективного значения через соотношение (16)

$\delta=\frac{\sqrt{U_{\mathrm{cp}} U_{\mathrm{M}}}-U_{9 \phi \phi}}{U_{9 \phi \phi}}=K-1$.

Отметим, что для определения величины $U_{\text {эфф }}^{2}$ через произведение среднего и амплитудного значений необходимо иметь вольтметр с линейным детектором, проградуированный в средних значениях (напр., Ф517) и вольтметр с пиковым детектором, проградуированный в амплитудных значениях
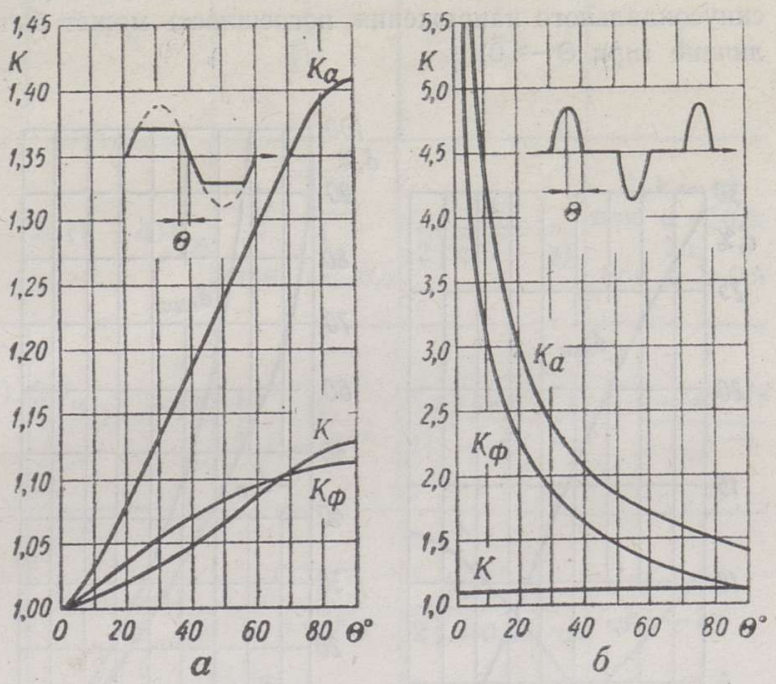

Рис. 1. (напр., B4-1A).

Введение новых определений и понятий буудет оправдано в том случае, если предложенный метод «вольтметров средних и амплитудных значений» имеет меньшую погрешность по сравнению с погрешностями, определяемыми выражениями (5) и (6).

Рассмотрим напряжения типа «синус с ограничением сверху» и жсинус с ограничением снизу» (рис. Іа и 1б). Заметим, что при $\theta=\frac{\pi}{2}$ имеем напряжение синусокдальной формы.

Среднее значение напряжений

$$
\begin{gathered}
U_{\mathrm{cp}}=\frac{2 U_{\mathrm{M}}}{\pi}\left[\frac{\pi}{2}-\theta+\frac{1}{\sin \theta}(1-\cos \theta)\right], \\
U_{\mathrm{cp}}=\frac{2 U_{\mathrm{M}}}{\pi} \frac{\sin \theta-\theta \cos \theta}{1-\cos \theta}
\end{gathered}
$$

(для рассматриваемых напряжений индекс «а» в нумерации формул относится к рис. $1 a$, индекс «б»-к рис. 16).

Эффективное значение напряжений

$$
\begin{aligned}
& U_{9 \phi \phi}=U_{M} \sqrt{\frac{2}{\pi}\left[\frac{\pi}{2}-\theta+\frac{1}{2 \sin ^{2} \theta}\left(\theta-\frac{1}{2} \sin 2 \theta\right)\right]} \\
& U_{9 \phi \phi}=\frac{U_{M}}{1-\cos \theta} \sqrt{\frac{2}{\pi}\left(\theta+\frac{\theta}{2} \cos 2 \theta-\frac{3}{4} \sin 2 \theta\right)} .
\end{aligned}
$$

Построив графики функций $K_{\phi}(\Theta), K_{a}(\Theta)$ и $K(\Theta)$, видим (рис. 1), что для напряжения типа «синус с ограничением снизу» функция $K(\Theta)$ относительно мало зависит от величины угла отсечки $\Theta$ (при изменении $\Theta$ от 0 до $90^{\circ} K(\Theta)$ изменяется от 1,118 до 1,128$)$, в то время как функции $K(\Theta)$ и $K_{\lambda}(\Theta)$, при $\Theta$ стремяшемся к нулю, стрежятся к бесконечности. Для напряжения типа «синус с ограничением сверху» функции 
$K_{\phi}(\Theta), K_{\mathrm{a}}(\Theta)$ и $K(\Theta)$ изменяются в конечных пределах. На рис. 2 приведены графики погрешностей $\delta_{\text {ср }}, \delta_{\text {пик }}$ и $\delta$. Из рис. 26 видим, что при измерении эффективного значения напряжения типа «синус с ограничением снизу» вольтметром с линейным детектором, проградуированным в эффективных вольтах синусоидального напряжения, c уменьшением угла $\Theta$ погрешность $\delta_{\text {ср }}$ стремится к величине - $100 \%$. При использовании вольтметра с пиковым детектором, проградуированным в эффективных значениях синусоидального напряжения, погрешность может быть равной бесконечно большой величине (при $\Theta \rightarrow 0$ ).
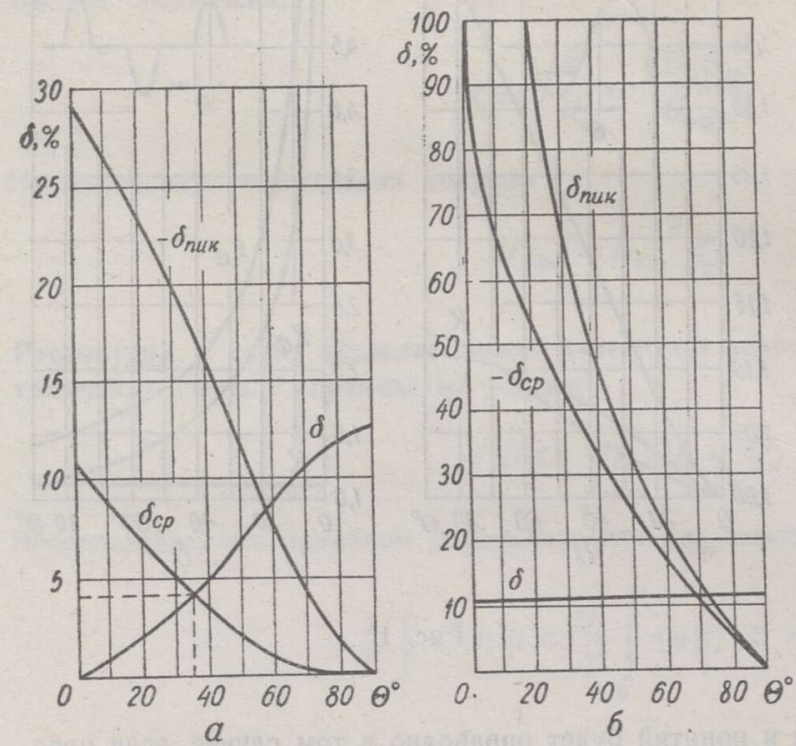

Рис. 2.

При использовании метода вольтметров средних и амплитудных значений, погрешность $\delta$ не превышает величины $12,8 \%$. Таким образом, в дапном случае метод вольтметров средних и амплитудных значений обеспечивает удовлетворительную для многих технических измерений точность во всем диапазоне возможных изменений угла отсечки $\Theta$.

Дальнейшее повышение точности измерения эффективного значения напряженнй возможно за счет комбинированного использования метода вольтметров средних и амплитудных значений и вольтметра с линейным детектором, проградуированного в эффективных вольтах синусоидального напряжения. Сказанное поясним на примере напряжения типа «синус с ограничением сверху». Из рис. $2 a$ видим, что при $\Theta=35 \div 90^{\circ}$ целесообразно использовать вольтметр с линейным детектором, проградуированный в эффективных значениях синусоидального напряжения, так как при этом $\delta_{c p}<\delta$ (практически шкала вольтметра средних значений должна иметь дополнительную градуировку в эффективных вольтах синусоидального напряжения). При $\Theta=0 \div 35^{\circ}$ бо́льшую точность измерения обеспечивает метод вольтметров средних и амплитудных значений. Считаем, что величина параметра $\Theta$ не известна. Снимаем остчет $U_{\text {эфф }}^{\prime}$ по шкале вольтметра средних значений, проградуи рованного в эффективных вольгах синусоидального напряжения (если дополнительная градуировка отсутствует, можно, например, использовать вольтметр В3-7). Затем определяем величину $U_{\text {эфф }}^{\prime \prime}$ методом вольгметров средних и амплитудных значений. Если при этом окажется, что $U_{\text {эфф }}^{\prime \prime}<U_{\text {эфф }}^{\prime}$ (в рассматриваемом случае $\left.\delta_{\text {ср }}>0\right)$ ), то бо́льшую точность обеспечивает метод вольтметров средних и амплитудных значений (мерой величины эффективного значения следует считать $U_{\text {эфф }}^{\prime \prime}$ ). Если $U_{\text {эфф }}^{\prime}<U_{\text {эфф }}^{\prime \prime}$, то мерой величины эффективного значения следует считать $U_{\text {эфф. }}^{\prime}$ В рассматриваемом случае комбинированный способ позволяет повысить точность измерения примерно в гри раза (максимально возможная погрешность $4,3 \%$ вместо 12,8\%, при использовании метода вольтметров средних и амплитудных значений).

При использовании комбинированного способа необходимо иметь предварительную информацию о знаке погрешности $\delta_{\text {cp }}$, что в конечном итоге сводится к определениг соотношения $K_{\phi}<K_{\phi 0}$ или $K_{\Phi}>K_{\phi 0}$.

Для проведения некоторых обобщений необходимо рассмстреть более широкий класс временных функций. В частности, согласно (12), для некоторых типов напря- 
Таблица 1

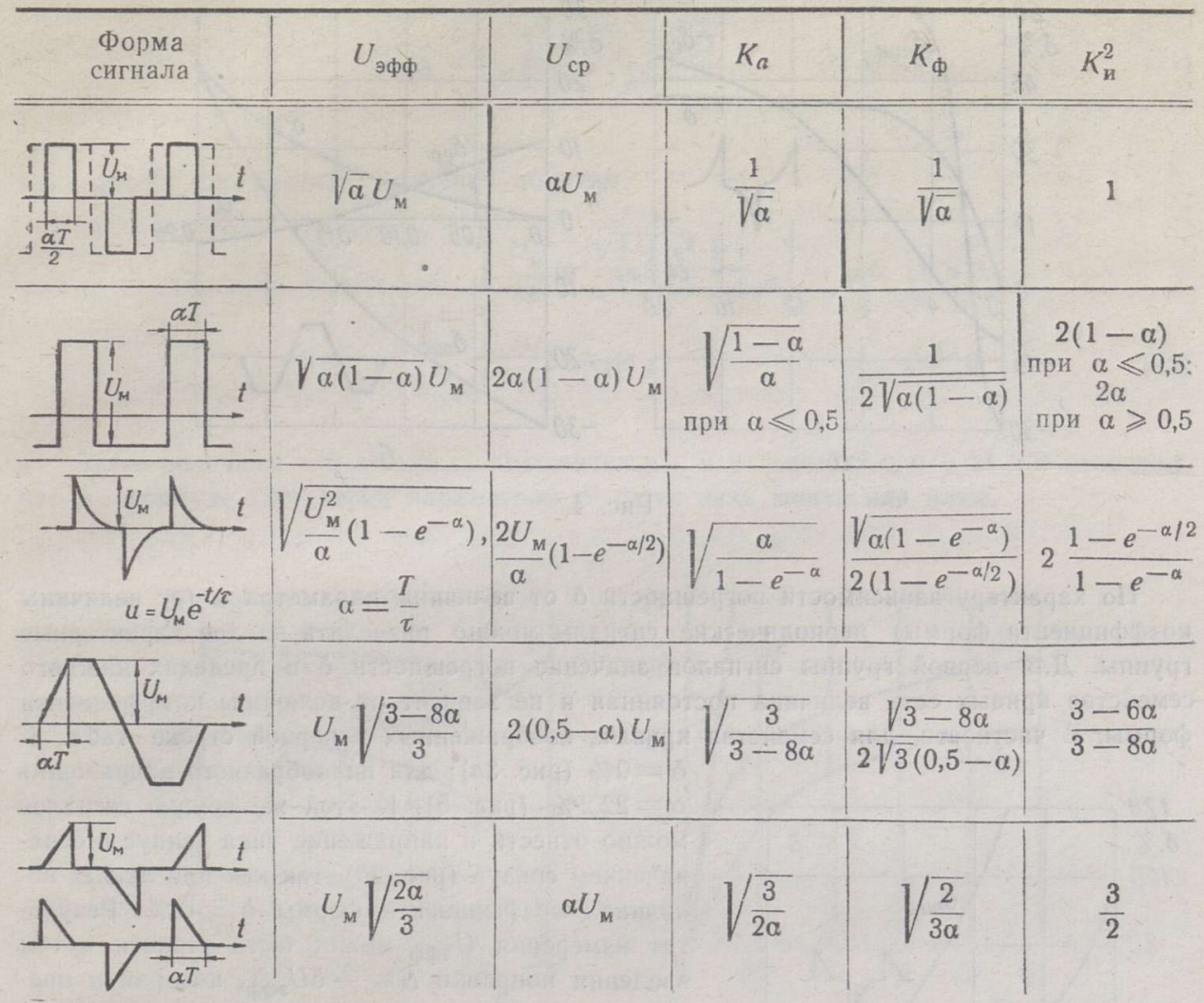

жения величина $K$ может быть равна единице, что соответствует строгому выполнению условия (16).

В табл. 1 приведены значения $U_{\text {эфф }}, U_{c p}, K_{\Phi}, K_{\mathrm{a}}$ и $K^{2}$ для некоторых типов периодических сигналов. Для семейств кривых, изображенных в первой строке таблицы. величина $K=1$, т. е. $\delta=0$ во всем диапазоне изменений параметра $\alpha$ (см. рис. $3 a$ ). Для остальных сигналов, изображенных в таблице, $K<\sqrt{2}$. Соответствующие графи. ки погрешностей изображены на рис. $3,4,5$.
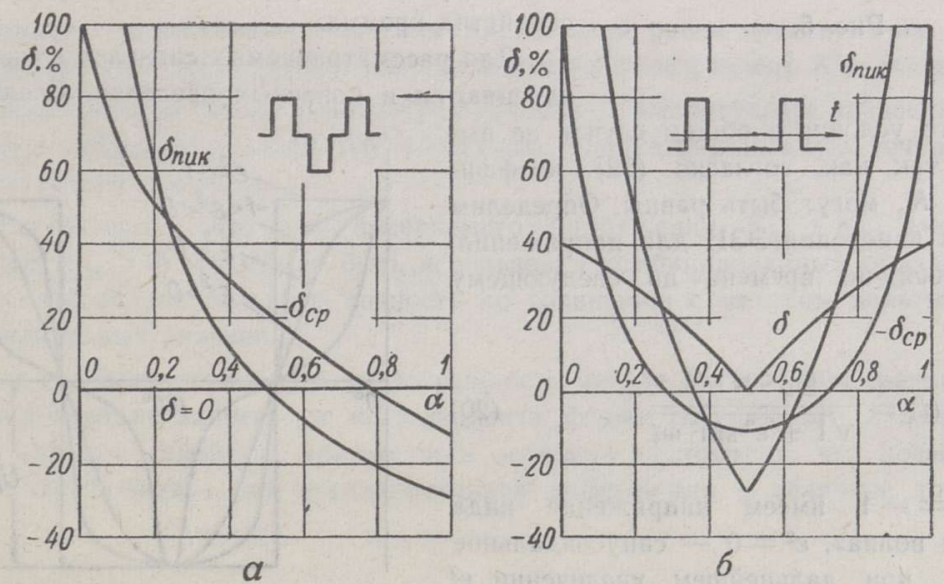

Рис. 3. 


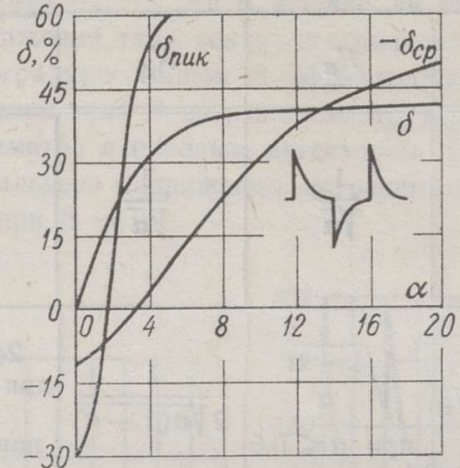

$\alpha$

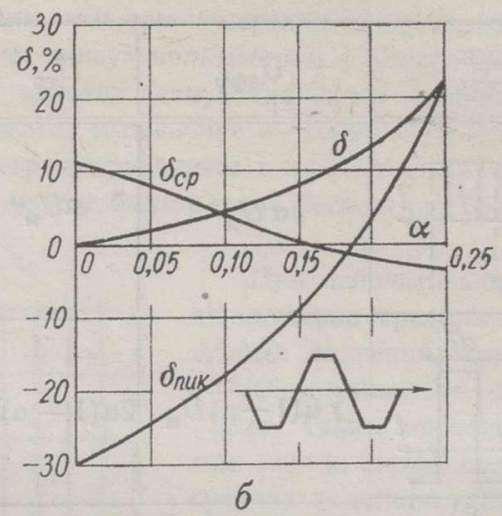

Рис. 4.

По характеру зависимости погрешности $\delta$ от величины параметра $\alpha$ (от величины коэффициента формы) периодические сигналы можно разделить на две характерные группы. Для первой группы сигналов значение погрешности $\delta$ в пределах каждого семейства кривых есть величина постоянная и не зависит от величины коэффициента формы. В частности, для семейства кривых, изображенных в первой строке табл. 1 ,

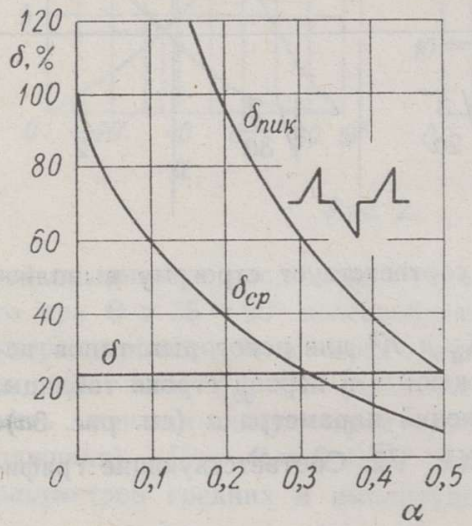

Рис. 5. $\delta=0 \%$ (рис. $3 a)$; для пилообразного напряжения $\delta=22,2 \%$ (рис. 5 ). К этой же группе сигналов можно отнести и напряжение типа «синус с ограничением снизу» (рис. 2б), так как при любых величинах коэффициента формы $\delta \approx 12 \%$. Резуль-

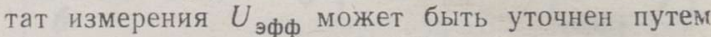
зведения поправки $\Delta=-\delta U_{\text {эфф}}$, которая в пределах каждого семейства кривых есть величина постоянная, независящая от величины коэффициента формы. Таким образом, для введения поправки отпадает необходимость иметь предварительную информацию о величине коэффициента формы, как это имеет место при измерении $Э 3 \mathrm{H}$ вольтметром с линейным детектором. Для второй группы сигналов величина погрешности $\delta$ зависит от коэффициента формы и в пределах каждого семейства кривых.

Для рассматриваемых сигналов функции $\delta(\alpha)$ изменяются в конечных пределах. Следует ожи-

дать, что это условие в общем случае не выполняется, так как, согласно (12), коэффнциенты $K$ и $K_{\text {a }}$ могут быть равны. Определим погрешность измерения $Э 3 Н$ для напряжения, изменяющегося во времени по следующему закону $\left[{ }^{4}\right]$ :

$$
u(t)=\frac{U_{\mathrm{M}} \cos \omega t}{\sqrt{1+\varepsilon^{2} \sin ^{2} \omega t}} .
$$

При $\varepsilon=-1$ имеем напряжение вида «квадратная волна», $\varepsilon^{2}=0$ - синусондальное напряжение, при дальнейшем увеличении $\boldsymbol{\varepsilon}^{2}$ кривая все больше заостряется (рис. 6). Для

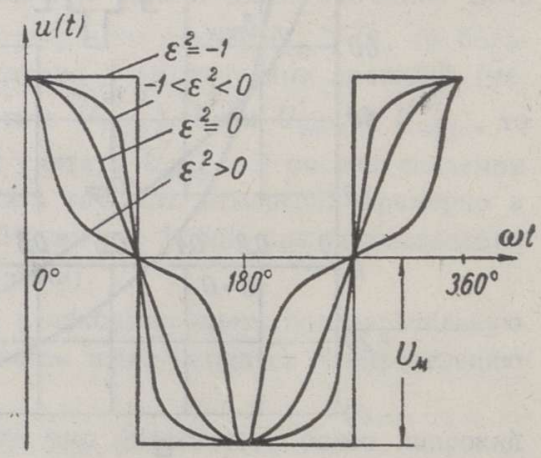

Рнс. 6. 
рассматрнваемой функцин

$$
U_{9 \phi \Phi}=U_{\text {м }} \sqrt{\frac{\sqrt{1+\varepsilon^{2}}-1}{\varepsilon^{2}}} \quad \text { при } \varepsilon^{2} \neq 0
$$

Согласно [5], для среднего значения получим

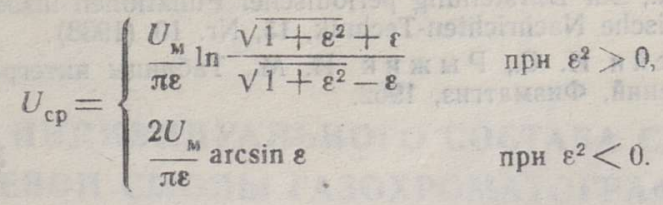

Здесь величнны $\varepsilon$ и $\varepsilon^{2}$ всегда положительны, а условия $\varepsilon^{2}<0$ и $\varepsilon^{2}>0$ означают, что в формуле $(20)$ перед параметром $\varepsilon^{2}$ стонт знак мннус или плюс.

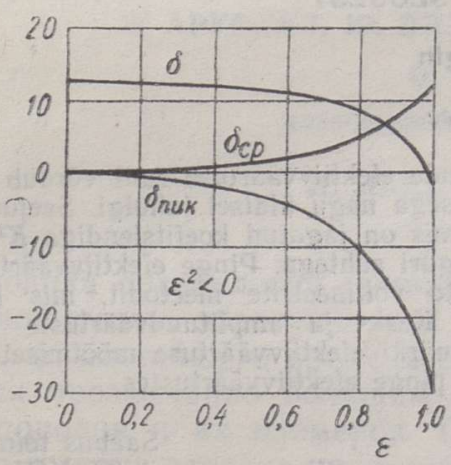

$\alpha$

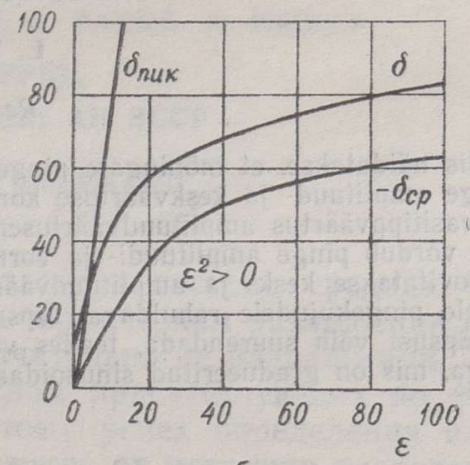

6

Pнс. 7.

На рнс. 7 приведены графики погрешностей $\delta_{\text {cD }}, \delta_{\text {пик }}$ и $\delta$. В данном случае для $\varepsilon^{2}>0$ величина погрешности $\delta$ больше погрешности $\delta_{c p}$ во всем диапазоне параметра $\varepsilon$.

Проведенный анализ позволяет сделать следующие выводы:

1. Квадрат эффективното значения напряжений равен произведению среднего и квазипикового (амплитудное значение, деленное на коэффициент $K^{2}$ ) значений.

2. Предложенный метод вольтметров средних и амплитудных значений для некөторых тинов напряжений позволяет обеспечнть удовлетворительную точность измерения эффективного значения.

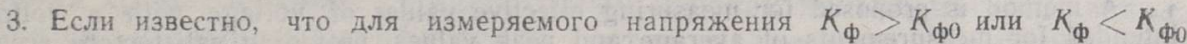
$\left(\delta_{\mathrm{cp}}<0\right.$ нли $\left.\delta_{\mathrm{cp}}>0\right)$, то может быть использован комбинированный способ измерения Э3Н, обеспечиваюший бо́льшую точность по сравнению с методом вольтметров средннх н амплитудных значений.

4. Для сигналов первой группы погрешность метода вольтметров средних и амплитудных значений не зависит от коэффициента формы измеряемого напряження, н $\mathbf{3}$ пределах каждого семейства кривых есть величнна постоянная, что позволяет получить значение поправки без предварительной информацин о величине коэффициента формы. 


\section{ЛИТЕ РАТ УРА}

1. D a vids o n J. J., Average vs RMS Meters for Measuring Noise, IRE Transactions on Audio, AU-9, No. 4, 108-111 (1961).

2. M a c doug a 11, J. S., Curves Find Value of Chopped Waveforms, Electronics, 32 No. $52,46,48$ (1959).

3. С м и рно в В. И., Курс высшей математики, ГИТТЛ, 1, 1953.

4. I o r d a n H., Zur Darstellung periodischer Funktionen insbesondere durch Bahnkurven, Elektrische Nachrichten-Technik, 15, Nr. 10 (1938).

5. Г р адштейн И. С., Рыжик И. М., Таблицы интегралов, сумм, рядов и про изведений, Физматгиз, 1962.

Поступила в редакцию

22. VIII 1963

\section{ELEKTRILISE SIGNAALI AMPLITUUD-, EFEKTIIV- JA KESKVAARTUSE VAHELISEST SEOSEST}

\section{Volgin \\ Resümee}

Artiklis näidatakse, et mõningate pingekujude efektiivväärtuse ruut võrdub ligikaudu sama pinge amplituud- ja keskväärtuse korrutisega nagu üldisel juhulgi. Seejuures määratakse kvasitippväärtus amplituudväärtusena, mis on jagatud koefitsiendiga $K^{2}$. Viimane omakorda võrdub pinge amplituudi- ja vormiteguri suhtega. Pinge efektiivväärtuse mõõtmiseks soovitatakse kesk- ja amplituudväärtuste voltmeetrite meetodit, mis kindlustab mõningatele pingekujudele rahuldava täpsuse. Kesk- ja amplituudväärtuste voltmeetri meetodi täpsust võib suurendada, teades vea märki efektiivväärtuse mõõtmisel lineaarse detektoriga, mis on gradueeritud sinusoidaalse pinge efektiivväärtustes.

Saabus toimetusse 22. VIII 1963

\section{ON THE INTERRELATION OF THE PEAK, EFFECTIVE AND AVERAGE VALUES OF THE ELECTRIC SIGNAL}

\section{Volgin}

\section{Sumnzary}

It has been stated that for certain types of voltage the square of effective value is approximately equal to the product of the peak and average values. In a general case, the square of the effective value is equal to the product of the average and quasi-peak values of the signal, the latter being determined as the peak value divided by the coefficient $K^{2}$, which equals the ratio of the amplitude and wave shape.

A method is proposed for measuring effective values of voltage with a voltmeter intended for measurements of average and peak values, providing satisfactory accuracy for certain types of voltage. The accuracy may be increased if information on the measurement error polarity is available, during measurements of effective values with a voltmeter having a linear detector calibrated in effective values of sinusoidal voltage. 